\title{
REALE UND IDEALE SCHICHTEN DES SPRACHKUNSTWERKS?
}

\section{Ein kritischer Beitrag zur Literaturontologie}

Die folgende Arbeit geht von der Überzeugung aus, daß die Frage nach dem genauen ontologischen Status literarischer Werke beantwortet werden muß, bevor wichtige und im eigentlichen Sinne literaturkritische Probleme in systematischer Weise auch nur gesehen, geschweige denn gelöst werden können. Dabei werden sich mehr oder weniger ausgedehnte Exkursionen in das Gebiet der Philosophie nicht vermeiden lassen: Grundsätzlichkeit in der Fragestellung auch literarischer Probleme fordert eben mit Notwendigkeit philosophische Entscheidungen.

Zum Problem der Seinsweise literarischer Gebilde existiert eine ansehnliche Literatur. Allerdings krankt diese Literatur an zwei bedeutenden Mängeln: erstens wurde sie zumeist von Fachphilosophen mit nur mäßigem literarischen Interesse und wohl auch bescheidener literarischer Bildung erstellt; zweitens widersprechen die ambitioniertesten Versuche dieser Richtung - also etwa die Roman Ingardens und Nicolai Hartmanns einander in entscheidenden Punkten. Die im Folgenden zu entwickelnde Auffassung widerspricht ihnen auch in wesentlichen Punkten, in denen sie untereinander einig scheinen. Umgekehrt weichen Untersuchungen, die von der Literaturwissenschaft herkommen, dem genannten Problem meist ganz aus, oder sie bringen es doch zu keiner befriedigenden Lösung. In diesem Zusammenhang muß man sogar die sonst mit Recht viel gerühmte „Theory of Literature" (1949) von René Wellek und Austin Warren kritisch anführen. In dieser Untersuchung wird die Frage nach dem Seinsstatus literarischer Werke zwar gestellt; ihre Beantwortung scheitert aber letztlich am Ungenügen des Begriffsapparats, der dafür bemüht wird. Wer, wie Wellek und Warren, nur ganz grob zwischen real, ideal und psychisch als möglichen Seinsweisen unterscheidet, wird allerdings zu dem wenig befriedigenden Ergebnis kommen müssen, literarische Werke seien keiner dieser Grundkategorien zuzuweisen; sie seien ,neither real, nor mental, nor ideal" (S. 144), sondern besäßen eben einen ,,special ontological status" (ebenda), über den allerdings, wie es scheint, nichts Genaueres ausgesagt werden kann. Der Grund für dieses negative Ergebnis scheint uns in einer allzu vereinfachten Auffassung des Idealen zu liegen. Mehrmals wird in dem genannten Band von Wellek und Warren dem literarischen Kunstwerk der Seinsstatus des Idealen mit der Begründung aberkannt, daß es dem Begriff „Dreieck” nicht vergleichbar sei. Dieser Feststellung ist zwar gewiß zuzustimmen; nur geht es nicht an, im sehr begrenzten Sonderfall des Dreieckbegriffs das ausschließliche und verbindliche Modell aller idealen Gegenstände zu sehen. Eine sorgfältigere Tren- 
nung der verschiedenen Arten idealer Seinsweisen hätte hier gewiß zu brauchbareren Ergebnissen geführt. Im folgenden Versuch machen wir uns zum Teil von Nicolai Hartmann (in „Das Problem des geistigen Seins”, 1933) getroffene Unterscheidungen zunutze, ohne uns jedoch seinen Ergebnissen immer anzuschließen.

Zunächst lassen sich, mit Hartmann, vier Seinsschichten tunlich voneinander unterscheiden: Anorganisches, Organisches, Seelisches und Geistiges. Die letztere, als die für uns interessanteste Schicht wird von Hartmann weiterhin dreifach untergeteilt in 1. personale, 2. objektive und 3 , objektivierte Grundform des Geistigen. Personaler Geist schließt dann alle individuellen Bewußtseinsakte ein; objektiver Geist deren historisch wirksamen Niederschlag in überdauernden Strukturen wie Sprache, Sitte, Recht, Kunst und Wissenschaft; objektivierter Geist endlich stellt, nach Hartmann, den Sonderfall eines an ein Realgebilde gebundenen geistigen Gehalts dar. Bei dieser Unterscheidung ist zu beachten, daß bei Hartmann den beiden ersten Grundformen, also dem personalen und objektiven Geist, voller Realcharakter zukommt; der dritten, sogenannten objektivierten Form wird solcher Realcharakter immerhin noch „zum Teil” zugeschrieben. Hier liegt allerdings ein Punkt, an dem wir Hartmann nicht zu folgen gedenken. Ähnlich wie Roman Ingarden und, an ihn gelehnt, Wellek und Warren, besteht nämlich Hartmann darauf, das Kunstwerk als ein Gebilde von ,vielschichtiger Struktur" und von ,doppelschichtiger Seinsweise" anzusprechen: einer "sinnlich-realen Vordergrundschicht" stehe eine „Hintergrundschicht geistigen Gehalts” gegenüber (wie oben, S. 364/5). Wie an anderer Stelle Ingarden, scheint uns hier auch Hartmann das Opfer einer Verwechslung zwischen dem Kunstwerk einerseits und seiner individuellen Kronkretisierung anderseits zu werden. Gewiß ist es der Zweck jedes Kunstwerks, nachvollzogen zu werden. Und ebenso gewiß ist die Vorbedingung für jeden solchen konkretisierenden Vollzug eine sinnlich-reale Basis. Diese Tatsachen sollten aber nicht darüber hinwegtäuschen, daß nur die Konkretisierung, nicht aber das Kunstwerk selbst, in ihrem Sein auf solche reale Basis angewiesen und daß dieses weder mit einem bestimmten Buchexemplar, noch mit dieser oder jener Aufführung oder Lesung zu identifizieren ist. Vielmehr handelt es sich beim Kunstwerk um ein Gebilde, dessen Entstehung zwar historisch fixierbar, das aber, einmal entstanden, durchaus unzerstörbar, transsubjektiv, zeit- und raumlos ist. Unzerstörbarkeit, Transsubjektivität, Zeitund Raumlosigkeit sind aber die Haupteigenschaften idealer Seinsweise. Auf Welleks und Warrens drei Grundkategorien zurückkommend, müßte man demnach sagen, psychisch ist der (persönlich-individuelle) Akt der Entstehung, wie auch der des Nachvollzugs, jedes Kunstwerks; real ist das Gebilde, in dem sich der Schöpfungsakt niederschlägt und durch das der Nachvollzug ermöglicht wird; ideal endlich ist das (rein intentionale) Gebilde des Kunstwerks selbst. Und von dem von Wellek und Warren so 
häufig bemühten idealen Dreieck unterscheidet sich dieses Kunstwerk somit nur in dreierlei Hinsicht: 1. durch seine größere Komplexität, 2. durch seine zeitlich fixierte Entstehung, 3. durch seine bemerkenswerte Eigenschaft, für den konkretisierenden Nachvollzug auf ein physisches (und damit selbst allerdings zerstörbares )Realgebilde angewiesen zu sein.

Mit dieser Ansicht stehen wir, wie gesagt, in sehr bewußtem Gegensatz nicht nur zu Nicolai Hartmann, sondern auch zu Roman Ingarden, der an entscheidender Stelle folgendermaßen formuliert:

„Die Schicht des literarischen Werkes, die sich aus Wortbedeutungen, Sätzen und Satzzusammenhängen aufbaut, hat kein autonomes ideales Sein, sondern ist auf ganz bestimmte subjektive Bewußtseinsoperationen, sowohl ihrem Entstehen wie auch ihrem Sein nach, relativ. Sie darf aber anderseits mit keinem konkret erlebten ,psychischen Inhalt" und auch mit gar keinem realen Sein identifiziert werden."

(,Das literarische Kunstwerk", S. 107)

Nun ist aber durchaus nicht einzusehen, weshalb ein Gebilde, das seinem Sein nach auf ,ganz bestimmte subjektive Bewußtseinsoperationen" angewiesen sein soll, umgekehrt mit keinem konkret erlebten psychischen Inhalt identifiziert werden dürfe. Ingarden kann hier seinen antipsychologistischen Absichten (die wir übrigens von Herzen teilen) selbst nicht treu bleiben, weil er das "Sein" des literarischen Werks unscharf faßt, indem er es von seinem konkreten Nachvollzug nur unzureichend unterscheidet - eine Verwechslung, die auch anderen Partien seines Buchs zum Schaden gereicht.

Dem gegenüber scheint es uns sowohl überzeugender als auch ökonomischer, das Sprachkunstwerk als eine rein ideale Struktur zu begreifen, ein Paradigma gewissermaßen, dessen Wesen darin besteht, implizite $D i$ rektiven für einen möglichen konkret-psychischen Nachvollzug zu erstellen. Die Tatsache, daß dieser Nachvollzug von Fall zu Fall im Detail verschieden ausfallen wird, ändert nichts an der grundsätzlichen Selbstindentität des Kunstwerks - die Direktiven, die sein Wesen ausmachen, sind durchaus unveränderlich, nur sind sie nicht erschöpfend. Vergleichbar wäre dieser Tatbestand etwa der Weise, in der man jemandem nahelegt, von einem Ort zu einem anderen zu wandern: die Beschreibung des Weges wird die wesentlichen Punkte fixieren, aber bei aller Konkretheit (dies ein wesentlicher Zug auch des Kunstwerks), nicht im Entferntesten jedes zu erwartende Detail aufzählen. Trotzdem läßt sich von einem besseren oder schlechteren, auch wohl von einem völlig verfehlten und umgekehrt von einem optimalen Befolgen der Direktiven sprechen. Auf das Sprachkunstwerk rückübertragen würde diese Parallele verdeutlichen, wie der ideale Direktivencharakter des einzelnen Werkes einerseits Raum läßt für individuelle Erlebnisweisen und Auslegungen, wie aber anderseits daraus keineswegs chaotische Willkür und relativistische Gleichwertigkeit einander widersprechender Deutungen folgen muß: Übereinstimmung über mehr oder weniger erfolgreiche Deutung, über ,,bessere” 
oder ,schlechtere” Erfassung eines Werks, ist im gleichen Maße zu erwarten, kann - mit Kant zu sprechen - vorgebildeten Lesern in ähnlicher Weise ,angesonnen" werden, wie Übereinstimmung in der Frage optimalen Folgens eines beschriebenen Wegs. Auch dem peinlichen Rekurs auf den Autor als einzige oder höchste Autorität in Deutungsfragen seines Werks wird durch diese Auffassung keineswegs Vorschub geleistet. Denn auch dem Autor sind ja die Direktiven, die sein Werk konstituieren, nicht erschöpfend gegenwärtig. Außerdem wandeln sich im Laufe der Geschichte, zwar nicht die Werke selbst, wohl aber die Möglichkeiten ihres optimalen Verständnisses und Nachvollzugs. Jeder Versuch von Selbstauslegung, wie interessant er auch an sich sein mag, muß sich deshalb gegen andere Auslegungen konkurrierend behaupten; und es bleibt denkbar, daß ein Interpret der Gesamtheit eines Werks kritisch besser gerecht wird als dessen Urheber.

Dieser Standpunkt, in Fragen der Interpretation zwischen Relativismus und absolutistischem Autoritätsglauben liegend, könnte man mit Wellek „Perspektivismus” nennen. Allerdings sollte man die Vaterschaft dieses Begriffs dann nicht nur bis Ortega y Gasset (von dem Wellek ihn übernommen haben will), sondern bis Nietzsche zurückverfolgen, dem Ortega y Gasset offensichtlich sowohl für das Wort, als auch die Konzeption und ihre philosophische Begründung weitgehend verpflichtet ist.*

Wird so der entweder undurchschaubare (Wellek und Warren) oder gemischte (Ingarden, Hartmann) ontologische Status des literarischen Kunstwerks zugunsten einer eindeutig idealen Bestimmung aufgegeben, so fallen damit auch die verschiedenen Schichtentheorien, wie sie Julius Kleiner, Waldemar Conrad, vor allem aber eben Ingarden und Hartmann entwickelt haben. Hartmann unterscheidet in seiner „Ästhetik" (1953) nicht weniger als sieben Schichten, die aus seinen ontologischen Grundkategorien nicht ohne Gewaltsamkeit entwickelt werden: als ,,mittlere Schichten". die Sphäre körperlicher Bewegung, Stellung, Mimik, Rede, 2. Handlungen und äußeres Verhalten: Aktionen und Reaktionen, 3. seelische Formung: Charakter und Eigenart der dargestellten Menschen, 4. Schicksal, das heißt das Ganze des Lebens der Charaktere; sowie, als sogenannte ,tiefe Schichten”, 5. individuelle Ideen, 6. das Allgemeinmenschliche und 7. Ideen, die nicht ausgesprochen werden, sondern erscheinen (op. cit., S. 177-182).

Es dürfte sich nach dem Gesagten erübrigen, auf diese reichlich buntscheckige Liste hartmannscher Schichten im Detail einzugehen. Das Hauptargument gegen Hartmanns Kollektion ist nicht, daß diese oder jene Schicht offensichtlich nicht konstitutiv genannt werden dürfte, auch nicht einmal, daß sich diese "Schichten" vielfach überschneiden,

* Zur Geschichte dieses Begriffs vgl. des Verfassers Aufsatz „Ortega y Gasset, Nietzsche and Modern Art" im Sammelband ,Man in Art", hg. M. Guiney, U. of North Carolina at Greensboro Symposium, 1963. 
sondern vielmehr, daß sich jene Liste beliebig verlängern ließe. Denn Hartmanns Auswahlprinzip scheint nicht mehr und nicht weniger zu sein als die Tatsache, daß was hier ,Schicht" genannt wird, nicht nur im realen Alltag, sondern auch im einen oder anderen Kunstwerk eine gewisse Rolle gespielt haben muß. Mit derselben Stringenz könnte man dann aber $z$. Bsp. eine Schicht der landschaftlichen, politischen oder beruflichen Umwelt postulieren, ferner eine des Wunschlebens der Charaktere, eine der Gefühle (untergeteilt etwa in individuelle und kollektive), eine des Allgemeinanimalischen etc.

Gewichtiger noch als solche Einwände ist aber die Tatsache, daß Hartmann mit seinen Schichten am Kunstwerk selbst, das heißt an all dem, was über ein realistisches Abkonterfeien menschlicher Schicksale hinausgeht, völlig vorbeisieht. $\mathrm{Daß}$ das Kunstwerk etwas ist, das gemacht wird, $\mathrm{da} ß$ es anderen Gesetzen gehorcht, als denen des faktischen Alltagsablaufs, $\mathrm{da} ß$ metaphorische, das heißt ,, uneigentliche”, aber umgekehrt in hohem Grade strukturierte Sprache ihr gemäßes Medium ist, daß für den Künstler alle der von Hartmann sogenannten ,Schichten" nicht mehr sind, als das Rohmaterial, aus dem ein Werk erst geschaffen werden kann - all dies sind Einsichten, die Hartmann entgangen, oder die hier doch völlig vernachlässigt sind: für den Philosophen scheint sich die Aufgabe der Kunst darin zu erschöpfen, daß sie gewisse Aspekte der realen Welt noch einmal präsentiert. Dadurch erübrigt es sich für Hartmann, speziell künstlerische Kategorien bzw. Schichten zu entwickeln. Stilistische Kunstmittel oder Kompositionsprinzipien wie das des Rhythmus, der Variation, der Steigerung, des Gegensatzes, der Verschränkung, der Raffung, der leitmotivischen Verweisung (um nur einige der wichtigsten zu nennen) haben in dieser Schichtenlehre deshalb keinen Ort, weil Hartmanns Schichten nicht im eigentlichen Sinn ästhetischen - geschweige denn literarischen - sondern rein fundamentalontologischen Überlegungen entspringen und von der realen Welt ohne Abstriche, Zusätze oder Änderungen auf die Welt des Kunstwerks übertragen werden. Dieselbe zutiefst kunstferne Perspektive ist denn auch verantwortlich für Hartmanns pauschal unkritische Überzeugung, daß in der Kunst allein der geschichtliche Rückblick zwischen echt und unecht zu unterscheiden vermöge, oder auch, daß nur das Epos, der Roman und das Drama die Bezeichnung „Dichtung großen Stiles" verdienten, die Lyrik aber dem gegenüber ,,keinen sehr hohen Anspruch" stelle (,Ästhetik”, 1953; S. 184).

Viel literaturvertrauter und, bei allem kritischen Vorbehalt, viel ernster zu nehmen sind denn auch Roman Ingardens vier bzw. fünf Schichten: Wortklang, Bedeutung, schematisierte Ansichten, Gegenstände und, als nicht-konstitutive Schicht, metaphysische Qualitäten. Wird, im Gegensatz zu Ingarden, der ideale Status des literarischen Kunstwerks als solchem erkannt, so folgt daraus freilich unter anderem, daß ontologisch die Schicht der Wortbedeutungen im weitesten Sinn, also denotativ und 
konotativ, die sogenannten anderen Schichten in der Tat, und zwar ohne Rest, subsummiert. Was Ingarden unter der Bezeichnung „Wortklang” eine eigene Schicht konstituieren läßt, erweist sich dann als - allerdings wichtiger - Aspekt individueller Konkretisierungen, nicht aber als Teil des Kunstwerks selbst. Schon das logische Grundkriterium der Selbstidentität des Werks muß ja so stark variierende Elemente wie den individuellen Sprechklang verschiedener Leser als Konstitutivum ausschließen; den Sprechklang etwa des Autors als verbindlich zu betrachten, scheint aber ebenso willkürlich, wie die Postulierung eines neutralisierten, sogenannten ,zeittypischen" Sprechklangs. Damit soll keineswegs geleugnet werden, daß der Sprachklang im Falle eines lyrischen Gedichts tatsächlich eine für die Wortwahl entscheidende und unvergleichbar wichtigere Rolle spielt als z. Bsp. in einem Roman. Nur muß hier genau unterschieden werden zwischen einem sogenannten ,potentiellen" Klang, der, ausdrücklich nicht ,real", selbst einen Aspekt der ideal-intentionalen Grundstruktur bildet und einem mit Recht ,real" genannten, physischen Klang, der aber von Lesung zu Lesung innerhalb gewisser Normen verschieden sein wird und deshalb nicht ein Konstitutivum des selbstidentischen Kunstwerks, sondern lediglich eine Seite seiner Konkretisierung darstellen kann.

Ingardens andere Schichten, die der „Gegenstände” und der „schematisierten Ansichten", sowie schließlich die enthusiastisch, aber etwas undeutlich apostrophierten „metaphysischen Qualitäten”, erweisen sich folgerichtig als bloße Perspektiven für die Auswahl der das Werk konstituierenden Bedeutungseinheiten - hier von ,Schichten" zu sprechen, die der Bedeutungsschicht ontologisch ebenbürtig zu sein hätten, scheint uns ganz abwegig. Zum Zwecke der methodischen Aufgliederung und praktischen Analyse könnte man allerdings (innerhalb der einen ontologischen Schicht) unterscheiden zwischen der in einem Sprachkunstwerk genannten gegenständlichen Welt und den in ihm implizierten Haltungen - das letztere wohl ein genaueres Wort für Ingardens „Ansichten”, die ja mit engl. „opinions" oder frz. ,avis" nichts zu tun haben.

Die relative Anwendbarkeit von Ingardens Kategorien in der literaturkritischen Praxis läßt vermuten, Ingardens - im Gegensatz zum sachfremden und systembefangenen Hartmann - verzeihlichere Schwäche habe ihren Grund darin, da $\beta$ er praktisch brauchbare Richtlinien über Gebühr hypostasierte und ein ontologisch genanntes Modell errichtete, in dem jede "Schicht" gewissermaßen das Echo eines an sich gültigen Aspekts kritischer Tätigkeit zu sein hatte. Alfred North Whitehead sprach in ähnlichen Fällen von einer ,fallacy of misplaced concreteness”.

Wir fassen zusammen. Es ging in der vorliegenden Arbeit darum, den ontologischen Status des Sprachkunstwerks zu bestimmen, die bekanntesten Schichtentheorien kritisch zu untersuchen und gewisse praktische Folgerungen aus dem vorgelegten Ansatz zu entwickeln. Im Gegen- 
satz zu Nicolai Hartmann und Roman Ingarden, in teilweisem Gegensatz auch zu René Wellek und Austin Warren, wurde das Sprachkunstwerk als eine intentionale Idealstruktur mit ausgesprochenem Direktivencharakter erkannt. Streng davon geschieden wurde von dieser selbstidentischen Gestalt der Akt des konkreten Nachvollzugs, der, von Fall zu Fall variierend, allein verdient, „real” genannt zu werden. Dem alten heuristischen Grundsatz, principia non sunt multiplicanda, folgend, wurde sodann der Versuch einer Prinzipienreduktion unternommen. Die verschiedenen von der Tradition sogenannten „Schichten" erwiesen sich dabei nicht als eigentliche (das heißt ontologisch ebenbürtige) Schichten, sondern als Auswahlprinzipien, die die ästhetische Struktur innerhalb der einen ontologischen Schicht mitbestimmen. Nicht zuletzt möchte die dargestellte Auffassung vom idealen Direktivencharakter des Sprachkunstwerks als Beitrag zur theoretischen Begründung einer kritischen Praxis verstanden werden, die in Fragen der Interpretation zwischen wertblindem Relativismus und gegenwerteblindem Absolutismus liegt und ,kritischer Perspektivismus” genannt werden könnte.

University of Michigan.

INGO SEIDLER.

\section{LES PREMIERS ROMANS WERTHERIENS FRANÇAIS: IMITATIONS OU PARODIES?}

Les ,,âmes sensibles" qui avaient versé des torrents de larmes à la lecture de la Nouvelle Héloise trouvèrent une nouvelle source d'émotion, une douzaine d'années plus tard. Cette fois, le livre vint d'outre-Rhin et acquit une telle popularité parmi les lecteurs bourgeois, se sentant peu appréciés ou même opprimés, qu'on le traduisit dix-huit fois jusqu'à la fin du siècle. „Il y avait dans la fièvre et la susceptibilité de Werther quelque chose d'analogue à leur propre inquiétude. Sa sentimentalité rêveuse le rendait sympathique au docile public des livres d'amour." 1 Ainsi le Werther de Goethe devint le premier ,,best-seller" allemand en France, et aida à préparer le terrain pour l'avènement de la génération romantique.

On ne se contenta pas de traductions. Il fallait des Werthers ,en habit français". Une lignée de jeunes auteurs, lecteurs fervents du roman de Goethe, plus enthousiastes que doués, entreprirent d'écrire leurs Werthers. Ils suivirent tous plus ou moins la trame fondamentale du modèle, la forme épistolaire, la prétention à l'authenticité; dans tous ces romans, ,la mélancolie causée par un amour interdit ou par une exaltation de la sensibilité se complique de l'amertume du roturier ou du petit bourgeois à qui la société refuse une place digne de sa valeur" (p. 20). Les versions vont de l'adaptation-traduction à l'imitation quasi-indépendente; elles n'omettent aucun trait extérieur de l'original et ajoutent des traits carac- 